\title{
Vínculos afectivos en la infancia y calidad en las relaciones de pareja de jóvenes adultos: el efecto mediador del apego actual
}

\author{
José L. Martínez-Álvarez*, Antonio Fuertes-Martín, Begoña Orgaz-Baz, Isabel Vicario-Molina y Eva González-Ortega
}

Facultad de Psicología. Universidad de Salamanca (España)

\begin{abstract}
Resumen: En este trabajo se persiguen dos grandes objetivos: a) conocer la asociación entre la vinculación afectiva con los padres en la infancia y la calidad percibida de las relaciones de pareja en la juventud; b) analizar el posible efecto mediador, en esa asociación, de la experiencia de apego adulto experimentado en la relación de pareja. En el estudio participaron de manera voluntaria 133 parejas heterosexuales (17-25 años). A través de un cuestionario on line, se evaluaron, en los dos miembros de la relación, la calidad de las relaciones con el padre y la madre en la infancia, el apego con la pareja actual (Ansiedad-Evitación), y la calidad percibida de la propia relación. Los resultados mostraron asociaciones significativamente positivas entre la vinculación afectiva en la infancia y la calidad de la relación, comprobándose además el efecto mediador del apego actual. Por último, se encontraron asociaciones negativas entre la ansiedad y evitación de cada miembro de la pareja y su propia experiencia relacional, y en algún caso, con la experiencia relacional del otro. Estos resultados se discuten en términos de la teoría del apego y su importancia de cara a la calidad de la relación de pareja.

Palabras clave: Apego; relación de pareja; apego adulto; calidad de la relación.
\end{abstract}

\section{Introducción}

El establecimiento y mantenimiento de relaciones afectivas e íntimas (o de pareja) en adolescentes y jóvenes se presenta como una tarea fundamental en su desarrollo psicosocial, con importantes implicaciones para la salud, bienestar y ajuste psicológico (Collins, 2003; Crouter y Booth, 2006; Furman y Shaffer, 2003). Aunque estas relaciones no están exentas de riesgos para el desarrollo de adolescentes y jóvenes (ver Furman, Ho y Low, 2008), cuando funcionan bien, además de ser importantes fuentes de apoyo social, emocional, instrumental o de ocio, contribuyen de manera fundamental a la elaboración y construcción de su identidad y a la mejora de la competencia social (Collins, Welsh, y Furman, 2009; Furman y Shaffer, 2003).

Uno de los factores claves para ese buen funcionamiento es la calidad de la relación. La calidad de la relación podemos definirla como el grado en que los miembros de la pareja muestran intimidad, afecto y apoyo mutuo (Collins et al., 2009). En general, las parejas que perciben una relación de pareja de alta calidad, manifiestan mayores niveles de bienestar, satisfacción, compromiso y relaciones futuras más positivas (Collins et al., 2009).

Sin embargo, y a pesar de los avances en esta línea, todavía no están totalmente definidos los factores intrapersonales e interpersonales que determinan esta calidad. La teoría del Apego, sin duda, se ha convertido en uno de los referentes más importantes, en este sentido (Holland, Fraley, y Roisman, 2012; Molero et al., 2011). Dos conceptos centrales

* Dirección para correspondencia [Correspondence address]: José L. Martínez Álvarez. Facultad de Psicología (Universidad de Salamanca). Ctra. Toro s/n, Salamanca 37005. E-mail: maral@usal.es
Title: Parental attachment in childhood and quality of the romantic relationships of young adults: the mediating effect of adult attachment Abstract: This paper has two main aims: firstly, to explore the relationship between parental attachment in childhood and perceived quality of romantic relationships in youth; secondly, to assess the possible mediating effect of adult attachment in the romantic relationship. A total of 133 heterosexual couples (ages 17-25) voluntarily participated in the study. An online questionnaire was applied to both partners to evaluate the quality of the relationships with the father and the mother in childhood, the attachment to partner (Anxiety-Avoidance), and the perceived quality of the romantic relationship. The results showed significant positive associations between parental attachment in childhood and the quality of the romantic relationship, which were mediated by the adult attachment to partner. Furthermore, negative associations were detected between anxiety and avoidance of each partner and their own relational experience (and the other's relational experience, in some cases). These results are discussed in terms of attachment theory and their significance to the quality of the romantic relationship.

Key words: Childhood attachment; romantic relationship; adult attachment; quality of the relationship.

subyacen a esta teoría: a) se entiende que la disponibilidad y el cuidado de las figuras de apego generan modelos mentales en el niño/a sobre sí mismos/as y los/las demás (en cuanto a expectativas, pensamientos, sentimientos y conductas) que les guían en la formación de relaciones futuras (Bowlby, 1969; 1982); b) Ainsworth, Blehar, Waters y Wall (1978), a partir de la conocida "situación del extraño", establecieron diferentes tipos de apego, y señalaron la importancia de la interacción entre la figura de apego y el niño como base segura para la exploración (explorar el ambiente con la confianza y seguridad que proporciona la figura de apego). Posteriormente, Ainsworth (1991) destacó el rol fundamental que el sistema de apego juega en las relaciones adultas, principalmente a partir de la base segura como un componente central de las mismas (si hay seguridad y disponibilidad en esas relaciones, el sujeto puede alejarse de esa base segura, con la confianza de poder comprometerse en otras actividades).

Pues bien, desde el planteamiento inicial y pionero de Bowlby (1969), el estudio del apego ha seguido dos líneas o trayectorias independientes (descrito en Mikulincer y Shaver, 2003): la relación padres-hijos durante la infancia y adolescencia y la de las dinámicas del apego en relaciones de pareja adultas. En relación a la infancia, según el modelo de Bowlby (1969; 1982), al que denominó el sistema de conductas de apego, ante situaciones amenazantes se activa el sistema de apego; si las figuras de apego se muestran disponibles y ofrecen la atención y cuidados necesarios, se va conformando un sistema de apego seguro. Si no se ofrece esa disponibilidad y atención, el apego será inseguro. En este caso podría adoptar dos formas: si la persona, de manera implícita o explícita, activa exageradamente el sistema de apego en las distintas situaciones que no percibe esa disponibilidad, se 
conforma un estilo de apego ansioso; en cambio, si inhibe o desactiva el sistema de apego, se conformará un estilo de apego evitativo. Lo deseable, por tanto, según esta teoría sería que las figuras de apego mostraran niveles altos de proximidad, cuidados, atención y disponibilidad para que la persona desarrollara un patrón de apego seguro.

Con el trabajo pionero de Hazan y Shaver (1987) surge el interés por el estudio del apego en las relaciones de pareja adultas. En él se defiende que estas relaciones comparten características similares a las experimentadas en la infancia entre el niño y sus cuidadores (p. e., sentimiento de seguridad y confianza ante la disponibilidad del otro, búsqueda frecuente del contacto íntimo y próximo, interés y preocupación por el otro, o sentimientos de inseguridad cuando el otro se muestra lejano e inaccesible). Posteriormente han sido numerosos los estudios que han incidido en las características de estas relaciones de apego y sus efectos en las relaciones de pareja tanto de adolescentes como de jóvenes y adultos (Feeney, 1999; Fraley, 2002; Heffernan, Fraley, Vicary, y Brumbaugh, 2012; Holland, Fraley, y Roisman, 2012; Mikulincer, Florian, Cowan y Cowan, 2002; Mikulincer y Shaver, 2003; Mikulincer y Shaver, 2007; Molero et al., 2011). Nuestro trabajo aborda precisamente la conexión entre el apego en la infancia, el apego en las relaciones de jóvenes adultos y la calidad de las mismas. Veamos algunos antecedentes.

\section{Apego y afecto con los padres, apego en las relacio- nes de pareja y calidad de las mismas}

La conexión entre el sistema de apego conformado en la infancia (a partir de las interacciones entre el niño y sus cuidadores) y las representaciones del apego en las relaciones adultas, es un tema recurrente en los desarrollos de la teoría del apego. Por ejemplo, Mikulincer y Shaver (2003; 2007) señalan que los modelos mentales generados en la infancia en las relaciones con las figuras de apego (padres y/o otros cuidadores), forman la base para las representaciones de apego a lo largo de la vida. Por su parte, Fraley (2002), en un meta-análisis, concluye que los patrones de apego se forman en las relaciones con los padres, y que estos patrones siguen ejerciendo una enorme influencia a lo largo del tiempo en las relaciones futuras. Más recientemente, Furman y Winkles (2010) encontraron que los estilos inseguros con los padres (evitativos y ansiosos) correlacionaron positivamente con estilos evitativos en las relaciones de pareja de jóvenes adolescentes, siendo los chicos quienes mostraron mayores niveles de estilo evitativo que las chicas.

Además, esta vinculación infantil también aparece asociada a la calidad de relaciones futuras. Los resultados de numerosos estudios señalan que cuanto más positivo es el ambiente familiar (caracterizado por la calidez parental, la estabilidad emocional, el apoyo o el afecto entre la pareja) mayor capacidad tendrá el/la joven para formar y mantener relaciones íntimas y mayor satisfacción experimentará en ellas (Auslander, Short, Succop, y Rosenthal, 2009; Collins y Dulmen, 2006; Donnellan, Larsen-Rife, y Conger, 2005;
Gray y Steinberg, 1999; Ha, Overbeek, de Greef, Scholte, y Engels, 2010; Leadbeater, Banister, Ellis, y Yeung, 2008; Mikulincer y Shaver, 2007).

Sin embargo, hay algunas cuestiones de interés que permanecen abiertas a la investigación científica. Una de ellas se refiere a las posibles diferencias en las experiencias de apego con el padre y la madre, y sus efectos tanto en los hijos como en las hijas. Así, parecen convincentes las pruebas sobre los patrones de socialización diferencial por parte de los padres hacia las hijas y los hijos, y cómo éstos se plasman en las interacciones cotidianas entre ellos (ver McHale, Crouter y Whiteman, 2003).

Pues bien, existen pocos indicios sobre estas diferencias y sus efectos en las relaciones de pareja posteriores, aunque suelen apuntar en dos direcciones, en ocasiones contradictorias: a) la relación con la madre, en general, tiene una mayor importancia que la relación con el padre de cara a la calidad de las relaciones futuras (Delgado, Oliva y Sánchez-Queija, 2011; Robinson, 2000; Seiffge-Krenke, Overbeek, y Vermulst, 2010); b) la importancia de la relación con el padre para las chicas y la relación con la madre en el caso de los chicos (Bucx y Seiffge-Krenke, 2010; Scharf y Mayseless, 2008).

Además de subrayar estas posibles diferencias, la mayoría de estudios muestran un efecto directo de las relaciones padres-hijos en las relaciones de pareja posteriores. Sin embargo, es posible que el apego conformado en las relaciones con los padres tenga un efecto también indirecto en las relaciones de pareja posteriores, es decir, que puedan existir importantes mediadores entre ambos. Así, Simpson, Collins, Tran y Haydon (2007) encontraron que el apego en la infancia predecía la calidad emocional en relaciones de pareja de jóvenes adultos. Sin embargo esta asociación estaba mediatizada por la competencia social en la infancia y las relaciones de apego seguras o inseguras con los amigos en la adolescencia. Por su parte, Scharf y Mayseless (2001) comprobaron que las relaciones padres-hijos se asociaron con la capacidad para intimar en adolescentes y jóvenes varones, aunque el efecto de esas relaciones estaba mediatizado por las capacidades socio-emocionales.

Por último, también contamos con numerosas pruebas sobre la conexión entre apego adulto y la calidad de las relaciones de pareja (Feeney, 1999; Gómez-Zapiain, Ortiz y Gómez-Lope, 2011; Holland, Fraley, y Roisman, 2012; Mikulincer, Florian, Cowan y Cowan, 2002; Mikulincer y Shaver, 2007; Molero et al., 2011). Concretamente, Mikulincer y colaboradores (Mikulincer et al., 2002; Mikulincer y Shaver, 2007) han señalado la importancia del apego específico a la relación, es decir, el que las personas van conformando en su relación concreta a partir de las experiencias e interacciones con su pareja. Se supone que en la medida que los dos miembros de la pareja sean fuentes de apoyo, seguridad e intimidad, y una base segura desde la que participar en actividades autónomas orientadas al desarrollo y crecimiento, mayor será la satisfacción de la relación. Pues bien, este apego ha resultado ser uno de los mejores predictores de la 
calidad de la relación (Lowyck, Luyten, Demyttenaere, y Corveleyn, 2008).

Sin embargo, también han aparecido algunas diferencias entre hombres y mujeres (ver Mikulincer y Shaver, 2007). En concreto, en esta revisión se señala que la ansiedad y evitación predicen la insatisfacción en la relación de las mujeres, mientras que la evitación lo hace principalmente en el caso de los hombres.

En resumen, a pesar del notable interés teórico y científico que ha despertado la teoría del apego y su conexión con las relaciones de pareja posteriores, todavía quedan cuestiones abiertas a la investigación. Por ejemplo, aunque hay muchas pruebas sobre las conexiones entre la vinculación afectiva en las relaciones con los padres y el establecimiento de relaciones de pareja en la juventud y vida adulta, nuevas evidencias señalan la importancia de determinados factores que pudieran actuar de mediadores (Scharf y Mayseless, 2001; Simpson, Collins, Tran y Haydon, 2007); tampoco sabemos con certeza en qué medida estos vínculos afectivos pueden estar asociados a la satisfacción y calidad de la relación de pareja, especialmente cuando valoramos posibles diferencias entre la vinculación afectiva a la madre y la vinculación afectiva al padre (Doyle, Lawford y Markiewicz, 2009). Por último, todavía son escasos los estudios que evalúan la experiencia del apego en términos diádicos, es decir, en ambos miembros de la relación de pareja (Molero et al., 2011), siendo más frecuente una evaluación individual del apego.

Pues bien, a partir de estas deficiencias, nos planteamos tres grandes objetivos en nuestro trabajo: 1) conocer la asociación entre vinculación afectiva con los padres en la infancia (apego) y la calidad de la relación de pareja en la juventud; 2) examinar la relación entre el apego adulto experimentado en la relación de pareja actual (ansiedad y evitación) y la calidad de esa relación; 3) comprobar el posible efecto mediador del apego adulto experimentado en la relación entre la vinculación afectiva con los padres y la calidad de la relación. Para que se diera este efecto mediador, y siguiendo los planteamientos de Baron y Kenny (1986) debería comprobarse una asociación directa entre apego hacia los padres, apego adulto y calidad de la relación; sin embargo la relación entre el apego hacia los padres y la calidad de la relación debería desaparecer cuando se controlan los efectos del apego adulto.

Teniendo en cuenta los antecedentes expuestos y las condiciones de mediación, nos planteamos las siguientes hipótesis: 1) si existe un mayor apego seguro a los padres en la infancia, entonces se darían menos niveles de apego ansioso y evitativo en la relación de pareja de jóvenes adultos (asociación negativa); 2) si se da un mayor apego seguro con los padres, especialmente la madre, entonces habrá una mayor calidad de la relación de pareja (asociación positiva). Podemos esperar también algunas diferencias entre los sexos: si hay un apego seguro al padre entonces la calidad percibida de la relación es mejor en las mujeres, en cambio, si hay un apego seguro a la madre entonces la calidad percibida de la relación será mejor en los hombres. 3) Si aumentan los nive- les de apego ansioso y evitativo en la relación de pareja, entonces disminuirá la calidad de esa relación (asociación negativa). 4) Como última prueba del proceso mediador, si tenemos en cuenta, conjuntamente, el nivel de apego ansioso y evitativo en la relación de pareja, entonces desaparecerá la asociación entre el apego seguro a los padres y la calidad de la relación de pareja (hipótesis 2). Esto probaría el efecto mediador del apego adulto.

\section{Método}

\section{Participantes}

En el estudio participaron 133 parejas heterosexuales. Las edades de los miembros de las parejas oscilaban entre los 17 y los 25 años, siendo la media de edad de los chicos $(M=20.36, D T=1.98)$ ligeramente superior a la de las chicas $(M=19.26, D T=1.74)$. La diferencia de edad entre los miembros de las parejas se situaba entre los 0 y los 6 años $(M=1.10, D T=1.58)$. En el $88 \%$ de las parejas, el chico era mayor que la chica con un rango de diferencia de edad entre los 0 y los 6 años. Únicamente en un 12\% de las parejas, la chica tenía más edad que el chico, y el rango oscilaba entre los 0 y 3 años. El tiempo medio que llevaban en la relación las parejas de nuestro estudio era de 2 años, siendo el mínimo de 2 meses y el máximo de 84 meses. La mediana se encontraba en los 20 meses.

En relación al nivel de estudios, la mayor parte de los varones señala estar cursando estudios universitarios (51.1\%), y en menor medida estudios de Bachillerato (14.2\%) o de Formación Profesional (14.2\%). El resto de los participantes masculinos $(20.6 \%)$ reconoce no cursar ningún estudio en la actualidad. En el caso de las mujeres, un $81.6 \%$ señala cursar estudios universitarios, un $15.6 \%$ estudios de Bachillerato, y un 2.1\% Formación Profesional. Sólo una de las participantes indicó no estar estudiando en la actualidad $(0.7 \%)$. Por último, la mayoría vivía con ambos padres (chicos: 90.2\%, chicas: $87.2 \%$ ) y un grupo reducido en una familia con un solo miembro (varones: $9.8 \%$, mujeres: $12.8 \%$ ). Ambos sexos tenían una media de 2 hermanos $(M=2.23, D T=0.95$, Mínimo $=1$, Máximo $=7$, en hombres; $M=2.06, D T=$ 0.72 , Mínimo $=1$, Máximo $=4$, en mujeres) .

\section{Procedimiento}

Los participantes del estudio fueron invitados a colaborar en una investigación sobre relaciones de pareja. El contacto se inició con estudiantes universitarios del área de las ciencias sociales (Psicología, Sociología y Trabajo Social). En concreto, al terminar una hora de clase de la universidad, un miembro del equipo investigador explicaba en el aula el interés y la necesidad de este tipo de estudios, y solicitaba a aquellas personas que estuvieran actualmente manteniendo una relación, su participación y la de su pareja. Además, se aclaraba que se iba a respetar su anonimato y el de sus parejas, y que la participación era voluntaria. Los participantes 
debían indicar su dirección de correo y la de su pareja, para posteriormente, recibir en su bandeja de correo electrónico un mensaje con un vínculo para responder a un cuestionario on-line. Aquellas personas que no contestaban en el plazo de quince días, recibían un segundo correo electrónico recordatorio. Sólo se tuvieron en cuenta las respuestas de aquellas parejas en las que ambos miembros habían contestado al cuestionario.

\section{Instrumentos}

Variables sociodemográficas. En este tipo de variables incluimos el sexo, edad, estructura familiar («tradicional» ambos padres- o monoparental), número de hermanos y posición en ellos, y nivel de estudios.

Vinculación afectiva con el padre y la madre en la infancia. Para valorar las conductas de apego en la infancia utilizamos el NRI-Behavioral Systems Version (NRI-BSV) debido a sus buenas propiedades psicométricas (Furman y Buhrmester, 2009). Este instrumento, frecuente en la investigación actual sobre el tema (Furman y Winkles, 2010), permite valorar en qué medida las relaciones de los adolescentes y jóvenes con sus padres, entre otras relaciones, se caracterizan por conductas asociadas con la activación de los sistemas de apego, cuidado y afiliación. En su totalidad, valora cinco características del apoyo social relacionadas con esos sistemas: a) el sujeto busca refugio seguro; b) el sujeto busca una base segura; c) el sujeto proporciona refugio seguro; d) el sujeto proporciona una base segura; y e) compañerismo. La adaptación consistió en valorar en qué medida los adolescentes y jóvenes percibían en los años infantiles a la madre y al padre como fuentes de base segura (de seguridad o confianza para permitir otras conductas de exploración) y refugio seguro (búsqueda de apoyo o confort en situaciones de ansiedad, alteración o preocupación), consideradas ambas claves en el sistema conductual del apego. Para ello utilizamos las subescalas Other Provides/Participant Seeks Secure Base (SSB) y Seeks Safe Haven (SSH). Cada una de ellas consta de 3 ítems, a los que se responde en una escala de 1 a 5 puntos. Para cada ítem se solicitaban dos respuestas, una encaminada a su relación con el padre y otra encaminada a su relación con la madre (ver Anexo I). La fiabilidad fue muy adecuada, tanto en hombres (Base Segura de la Madre "alfa" = .81; Base Segura del Padre, "alfa" =.94; Refugio de la Madre, "alfa" = .93; Refugio del Padre, "alfa" = .95), como en mujeres (Base Segura de la Madre "alfa" = .86; Base Segura del Padre, "alfa" =.87; Refugio de la Madre, "alfa" = .91; Refugio del Padre, "alfa" $=.92)$.

Apego con la pareja. Para la valoración del apego adulto, utilizamos una adaptación española (Alonso-Arbiol, Balluerka y Shaver, 2007) del Experiences in Close Relationships-Revised (ECR-R, Fraley, Waller y Brennan, 2000). Es uno de los más utilizados actualmente en el estudio del apego (Holland, Fraley, y Roisman, 2012), generalizándolo incluso a otro tipo de relaciones (Fraley, Heffernan, Vicary, y Brumbaugh, (2011). El cuestionario cuenta con 36 ítems que valoran las dos di- mensiones básicas relacionadas con el Apego: Ansiedad, es decir el grado de seguridad e inseguridad respecto a la disponibilidad y respuesta del otro, y Evitación, es decir el grado de comodidad o incomodidad experimentado en relación con la proximidad y dependencia del otro (ver una muestra de ítems en Anexo II). La fiabilidad alfa de Cronbach fue de .85 para la Ansiedad y de .78 para la Evitación en el caso de los hombres, y de .87 y .70, respectivamente, en el caso de las mujeres.

Calidad de la relación. Se evaluó a través de una adaptación de los cuatro ítems utilizados por Conger, Cui, Bryant y Elder (2000) que valoran el grado de felicidad, satisfacción y compromiso en la relación de pareja. Todos los ítems se presentan en una escala tipo Likert de 5 puntos. La fiabilidad alfa de Cronbach fue de .75 en el caso de los hombres, y de .78 en el caso de las mujeres.

\section{Resultados}

\section{Estrategia de análisis}

Para la primera condición de mediación se supone que tiene que haber una conexión entre la vinculación afectiva con los padres en la infancia y el apego experimentado en la relación de pareja actual. Para comprobar esta condición realizamos análisis de regresión simple entre los factores asociados con la vinculación afectiva con los padres en la infancia (Base Segura del Padre -BSP-, Base Segura de la Madre BSM-y Refugio Seguro del Padre -RSP- y Refugio Seguro de la Madre -RSM) y la Ansiedad-Evitación en la relación de pareja actual.

La segunda condición de mediación exige una asociación entre la vinculación afectiva con los padres, la vinculación afectiva con la pareja y la calidad de la relación de pareja. Para comprobarlo calculamos las regresiones simples entre los dos grupos de factores anteriores con la calidad de la relación de pareja actual.

Para probar la tercera condición de mediación, y siguiendo los planteamientos de Baron y Kenny (1986), realizamos análisis de regresión jerárquica con el fin de comprobar en qué medida la calidad de la relación de pareja actual era explicada por la experiencia afectiva percibida hacia los padres, por la Ansiedad-Evitación propia o por la de la pareja. Para ello, incluimos únicamente aquellas variables que en los análisis de regresión simples contribuían significativamente a explicar la calidad de la relación de pareja actual. En un primer paso, incluimos aquellas variables relacionadas con la vinculación con los padres; en el segundo, las relacionadas con la Ansiedad-Evitación propias; y, en el último, las relacionadas con la Ansiedad-Evitación de la pareja.

Finalmente, planteamos análisis de regresión jerárquica por pasos sucesivos, de forma que nos permitiera obtener aquellas variables realmente relevantes de cada uno de los tres bloques para explicar la calidad de la relación de pareja actual. Si existe mediación, la significación de las variables afectivas con los padres en el primer paso, debe desaparecer 
en el segundo y tercer paso. Todos estos análisis los realizamos por separado para la calidad de la relación percibida por los hombres y las mujeres de las parejas. Los análisis los llevamos a cabo con el paquete estadístico SPSS 18 (2010) considerando niveles de significación inferiores a .05 .

\section{Vinculación afectiva en la infancia y en la relación de pareja adulta}

En los dos análisis de regresión simple (hombres y mujeres) la Ansiedad y la Evitación fueron consideradas las variables criterio, y la BSP, la BSM, el RP y el RM fueron consideradas las variables predictoras. Como se puede observar en la Tabla 1, en el caso de los hombres el RM estaba negativamente asociado con la Ansiedad en la relación de pareja. Además, todas las variables -BSP, BSM, RP y RM- estaban significativamente asociadas de manera negativa con la Evitación.

En el caso de las mujeres, el Refugio, tanto al padre como a la madre, está negativamente asociado con la Ansiedad en la relación de pareja. En relación a la Evitación, sólo está asociada significativa y negativamente con la BSP.

En definitiva, estas asociaciones, en su conjunto, corroboran la primera condición en la mediación, que establece algún tipo de conexión significativa entre la vinculación afectiva en la infancia y en las relaciones adultas.

\section{Ansiedad-Evitación adulto y calidad de la relación de pareja}

La segunda condición trata de probar la asociación entre la Ansiedad y Evitación en la relación de pareja y la calidad de la misma. En este caso, en los análisis de regresión simple (uno para cada miembro de la pareja), la Ansiedad y la Evitación (tanto propias como de la pareja) fueron consideradas las variables predictoras, y la calidad de la relación la variable criterio. Como puede observarse en la tabla 1, en todos los casos encontramos asociaciones significativamente negativas; es decir, a niveles más elevados de Ansiedad y Evitación menor Calidad percibida de la Relación de pareja actual. Estos resultados avalarían la segunda condición de la mediación.

Además, nos estarían indicando que los apegos inseguros en la relación de pareja (Ansiedad y Evitación) se asocian con una percepción de la relación de pareja de menor calidad, en términos de satisfacción, compromiso y ajuste. Igualmente, aunque los apegos inseguros del otro miembro de la pareja se asocian también con una menor calidad de la relación de pareja experimentada, en el caso de las mujeres, la Ansiedad del hombre juega un rol muy importante en su experiencia de menor calidad de la relación.

Tabla 1. Pesos beta y coeficientes de determinación de las regresiones simples tomando como variables criterios Ansiedad, Evitación y Calidad de la relación.

\begin{tabular}{|c|c|c|c|c|c|c|}
\hline \multirow[b]{3}{*}{ V. Predictoras } & \multicolumn{3}{|c|}{ V. Criterio } & \multicolumn{3}{|c|}{ V. Criterio } \\
\hline & \multicolumn{3}{|c|}{ Hombres } & \multicolumn{3}{|c|}{ Mujeres } \\
\hline & Ansiedad & Evitación & Calidad & Ansiedad & Evitación & Calidad \\
\hline
\end{tabular}

\begin{tabular}{|c|c|c|c|c|c|c|c|c|c|c|c|c|}
\hline & $\beta$ & $\mathrm{R}^{2}$ & $\beta$ & $\mathrm{R}^{2}$ & $\beta$ & $R^{2}$ & $\beta$ & $\mathrm{R}^{2}$ & $\beta$ & $\mathrm{R}^{2}$ & $\beta$ & $R^{2}$ \\
\hline Base Segura Padre & -.06 & .00 & $-.17 *$ & .02 & .16 & .02 & -.16 & .02 & $-.26 * *$ & .06 & $.24 * *$ & .05 \\
\hline Base Segura Madre & -.08 & .00 & $-.18^{*}$ & .02 & $.23^{* *}$ & .04 & -.16 & .02 & -.00 & .00 & $.21 *$ & .04 \\
\hline Refugio Padre & -.15 & .02 & $-.23 * *$ & .04 & .16 & .02 & $-.21 *$ & .04 & -.16 & .02 & $.20 *$ & .04 \\
\hline Refugio Madre & $-.22^{*}$ & .04 & $-.29 * * *$ & .08 & $.24 * *$ & .05 & $-.18^{*}$ & .02 & -.03 & .00 & .11 & .01 \\
\hline Ansiedad Propia & & & & & $-.40^{* * *}$ & .15 & & & & & $-.27 * *$ & .07 \\
\hline Evitación Propia & $\left(.33^{* * *}\right)^{1}$ & & & & $-.58 * * *$ & .32 & $\left(.17^{*}\right)$ & & & & $-.50 * * *$ & .25 \\
\hline Ansiedad Pareja & $\left(.34^{* * *}\right)$ & & $(.24 * *)$ & & $-.26 * *$ & .06 & $(.34 * * *)$ & & $\left(.18^{*}\right)$ & & $-.42 * * *$ & .17 \\
\hline Evitación Pareja & $\left(.18^{*}\right)$ & & $(.22 * *)$ & & $-.21 *$ & .04 & $(.24 * *)$ & & $(.22 *)$ & & $-.29 * * *$ & .08 \\
\hline
\end{tabular}

${ }^{*} p<.05, * * p<.01, * * * p<.001$

${ }^{1}$ Entre 0 : correlaciones entre las variables ansiedad-evitación propias y de la pareja.

Efecto mediador de la Ansiedad-Evitación entre la vinculación afectiva en la infancia y la calidad de la relación de pareja

En un primer momento nos interesa conocer en qué medida la calidad de la relación de pareja actual es explicada por la experiencia afectiva hacia los padres, por la AnsiedadEvitación propia o por la Ansiedad-Evitación de la pareja. En el Análisis de Regresión Jerárquica encontramos: a) tanto en los varones $\left(R^{2}=.32\right)$ como en las mujeres $\left(R^{2}=.25\right)$ de las parejas, la Calidad de la Relación es explicada en mayor medida por la Ansiedad y Evitación propias que por las de la pareja, sobre todo en el caso de los varones; b) que los fac- tores relacionados con la vinculación afectiva con los padres en la infancia, explican un $8 \%$ de la varianza; y c) que la Ansiedad-Evitación de la pareja contribuye significativamente a explicar la Calidad de la Relación en el caso de las mujeres $\left(\mathrm{R}^{2}=.08\right)$; pero no, en el de los hombres $\left(\mathrm{R}^{2}=.01\right)$.

Por último, los resultados obtenidos en la Regresión Jerárquica Paso a Paso, nos permiten plantear modelos explicativos de la calidad percibida de la relación diferentes para los varones y las mujeres de las parejas de nuestro estudio.

En el caso de los hombres (Tabla 2), de los dos factores relacionados con la vinculación afectiva con los padres que se relacionaban significativamente con la Calidad de la Relación (Base segura y Refugio de la Madre), cuando se inclu- 
yen los dos en el primer paso, únicamente el Refugio de la Madre contribuye de forma significativa; cuando en el segundo paso se incluye la Ansiedad y la Evitación, las dos contribuyen significativamente, pero es la evitación la que explica en mayor proporción, y la contribución del Refugio de la Madre ya no es estadísticamente significativa (como hemos visto previamente, la relación entre Refugio de la Madre y Evitación o Ansiedad era estadísticamente significativa). Por lo tanto, el modelo final que explica la Calidad de la Relación percibida por los varones de las parejas incluye la Ansiedad y la Evitación propias y el Refugio de la Madre, aunque el efecto de esta última queda mediado por las variables Ansiedad y Evitación $\left[F(3,129)=26.46, p<.001, R^{2}\right.$ $=.38]$.

Tabla 2. Regresión jerárquica paso a paso en la que se explica la calidad de la relación de pareja en función de variables relacionadas con la vinculación afectiva con los padres, la ansiedad-evitación propia y la de la pareja en el caso de los varones.

\begin{tabular}{|c|c|c|c|}
\hline \multirow[b]{2}{*}{$\begin{array}{l}\text { Regresión } \\
\text { jerárquica }\end{array}$} & \multicolumn{3}{|c|}{ Hombres } \\
\hline & Regresión paso a paso & $\beta$ & $\mathrm{R}^{2}$ \\
\hline $1^{\circ}$ Paso: & Refugio de la Madre & $.24^{* *}$ & .06 \\
\hline $2^{\circ}$ Paso: & $\begin{array}{l}\text { Refugio de la Madre } \\
\text { Evitación Propia }\end{array}$ & $\begin{array}{l}.08 \\
-.55^{* * *}\end{array}$ & .28 \\
\hline & $\begin{array}{l}\text { Refugio de la Madre } \\
\text { Evitación Propia } \\
\text { Ansiedad Propia }\end{array}$ & $\begin{array}{l}.05 \\
-.49 * * * \\
-.23 * *\end{array}$ & .04 \\
\hline
\end{tabular}

En el caso de las mujeres (Tabla 3), cuando se incluyen en el primer paso los tres factores vinculados a los padres que se relacionaban significativamente con la Calidad de la Relación (Base segura del padre y de la madre y Refugio del Padre), únicamente la Base Segura del padre contribuye de forma significativa; cuando en el segundo paso se incluyen la Ansiedad y Evitación propios, es la evitación la que explica en mayor proporción la Calidad, mientras que la contribución de la Base Segura del padre deja de ser estadísticamente significativa (como hemos visto previamente la relación entre Base Segura del Padre y Evitación era significativa). Y, por último, en relación con la ansiedad-evitación de la pareja, en el siguiente paso se incluye la ansiedad de la pareja, y deja de ser significativa la ansiedad propia, como se puede observar la relación entre la ansiedad propia y la de la pareja es muy elevada $(r=.34, p<.001)$ y la relación con la Calidad Percibida de la relación es más elevada la de la ansiedad de la pareja que la propia (Ver Tabla 1). El modelo final que explica la Calidad de la Relación percibida por las mujeres de las parejas incluye la Evitación y Ansiedad propias; además del efecto de la Base Segura del Padre mediado por la Evitación propia, y de la Ansiedad Propia mediado por la Ansiedad de la Pareja $\left[F(4,128)=19.31, p<.001, R^{2}=.38\right]$.
Tabla 3. Regresión jerárquica paso a paso en la que se explica la calidad de la relación de pareja en función de variables relacionadas con la vinculación afectiva con los padres, la Ansiedad-Evitación propia y la de la pareja en el caso de las mujeres.

\begin{tabular}{|c|c|c|c|}
\hline \multirow[b]{2}{*}{$\begin{array}{l}\text { Regresión } \\
\text { jerárquica }\end{array}$} & \multicolumn{3}{|c|}{ Mujeres } \\
\hline & Regresión paso a paso & $\beta$ & $\mathrm{R}^{2}$ \\
\hline $1^{\circ}$ Paso: & Base segura padre & $.24 * *$ & .06 \\
\hline \multirow[t]{5}{*}{$2^{\circ}$ Paso: } & Base segura padre & .11 & \\
\hline & Evitación propia & $-.48 * * *$ & .21 \\
\hline & Base segura padre & .08 & \\
\hline & Evitación propia & $-.45^{* * *}$ & .03 \\
\hline & Ansiedad propia & $-.18^{*}$ & \\
\hline \multirow[t]{4}{*}{$3^{\circ}$ Paso: } & Base segura padre & .08 & \\
\hline & Evitación propia & $-.42 * * *$ & .08 \\
\hline & Ansiedad propia & -.08 & \\
\hline & Ansiedad pareja & $-.30 * * *$ & \\
\hline
\end{tabular}

\section{Discusión}

En este trabajo tratamos de comprobar la importancia que tiene la vinculación afectiva con los padres a la hora de explicar la calidad experimentada en la relación de pareja actual. Además, intentamos probar si estos efectos eran directos o bien indirectos, es decir, mediatizados por otros procesos afectivos como son la Ansiedad-Evitación percibida en la relación de pareja. En general, los datos se acomodan a nuestras hipótesis aunque también aparecieron algunas cuestiones de interés no esperadas.

De manera global, podemos decir que encontramos una asociación negativa entre la vinculación afectiva en la infancia y la Ansiedad-Evitación en la relación de pareja actual, aunque con algunas diferencias entre los sexos. Así, la Ansiedad estaba asociada negativamente con el Refugio de la Madre en ambos sexos, y en las mujeres, lo estaba también con el Refugio al Padre. Es decir, en la medida que las mujeres percibían a los padres y a las madres como fuentes de seguridad, apoyo,... en situaciones de ansiedad y preocupación, menores niveles de Ansiedad mostraron en sus relaciones de pareja. En cambio, en los hombres sólo era relevante la percepción que tenían de sus madres como fuentes de seguridad y apoyo.

En el caso de la Evitación por parte de los hombres, todas las variables se asociaban negativamente con ella, mientras que en las mujeres sólo lo hacia la Base Segura del Padre. Por lo tanto, podemos pensar que para ellos, cuanto mayor es la vinculación afectiva en la infancia en términos de apego con ambos padres (fuentes de seguridad, confianza, confort, apoyo,...), menor es el grado de Evitación que manifiestan en su relación de pareja. Sin embargo, para ellas, es sólo la seguridad proporcionada por el padre en la infancia la que se corresponde con una mayor comodidad en la 
proximidad y dependencia del otro en su relación de pareja actual.

En definitiva, nuestros datos confirman esa conexión entre el sistema de apego conformado en la infancia en las relaciones con los padres y las representaciones del mismo en las relaciones de pareja posteriores (Furman y Winkles, 2010; Mikulincer y Shaver, 2003; 2007). No obstante, nuestros datos parecen indicar que el padre del sexo opuesto juega un rol más significativo que el del propio sexo, aunque, en aspectos diferentes de la relación de pareja: en los chicos, el apoyo proporcionado por la madre especialmente parece importante para la percepción de seguridad y disponibilidad de su pareja actual, mientras que en las chicas, la seguridad y confianza del padre lo sería para la percepción de comodidad ante situaciones de proximidad e intimidad.

Por lo tanto, estos resultados vendrían a confirmar una vez más que seguramente los modelos mentales generados en la infancia con las figuras de apego tienen importantes consecuencias en las relaciones de pareja posteriores (Mikulincer y Shaver, 2003; 2007). Sin embargo, también es probable que los procesos de vinculación afectiva puedan variar en importancia en función del rol de padre-madre y el sexo del hijo. Nuestros datos apuntan hacia una mayor particularidad de las díadas padre-hija y madre-hijo en algunos efectos posteriores.

Algunos trabajos ya han incidido en la importancia del apego a la madre a la hora de explicar la calidad de la relación de pareja, aunque no han establecido diferencias entre hijos e hijas (Doyle, Lawford y Markiewicz, 2009), pero otros ya han avalado estas diferencias. Por ejemplo, (Bucx y SeiffgeKrenke, 2010) encontraron que las habilidades de resolución de conflicto en parejas adolescentes estaban asociadas a la calidad del apego con el padre en el caso de las chicas, y a la calidad del apego con la madre en el caso de los chicos. De manera similar, Scharf y Mayseless (2008) en un estudio con chicas en la última etapa adolescente, comprobaron que la calidad de la relación con la madre estaba asociada con el retraso en el inicio de las relaciones de pareja, mientras que la mejor calidad de la relación con el padre estaba asociada con la mejor calidad de la relación de pareja una vez que esta ya estaba formada.

Es posible que en la configuración de la identidad interpersonal (capacidad de intimar, sentirse próximo a otros,...) la importancia del contexto familiar, y especialmente la madre, sea más relevante para los hombres en comparación a las mujeres (se supone que tendrían menos dificultades en este campo). Sin embargo, en el caso de las mujeres la seguridad proporcionada por el padre también parece importante a la hora sentirse bien en situaciones de intimidad y proximidad en su relación de pareja. Es posible que el desarrollo óptimo requiera un ajuste y equilibrio entre autonomíaindividuación y conexión-vinculación. Si asumimos que los hombres estarían más orientados en la primera dimensión y las mujeres en la segunda, la vinculación con el progenitor del sexo opuesto contrabalancea esa orientación. De cualquier forma, nuestros resultados destacan la importancia de distinguir la vinculación afectiva con el padre y con la madre (Doyle, Lawford y Markiewicz, 2009). No cabe duda que serían aspectos a tener en cuenta en trabajos futuros.

El segundo objetivo trataba de probar la asociación entre la Ansiedad-Evitación en la relación de pareja actual y la calidad de la misma. En este caso, nuestra hipótesis también se vio confirmada: la inseguridad percibida en la relación en términos de ansiedad y evitación, se asociaba con una menor calidad en términos de satisfacción, compromiso, etc. en ambos sexos. Además, en el caso de las mujeres, la experiencia de mayor ansiedad por parte de sus parejas, se asocia con una menor calidad percibida en ellas. Es decir, en la medida que ellos experimentan mayores niveles de inseguridad respecto a la disponibilidad de su pareja, menores niveles de calidad de la relación perciben ellas.

Dos conclusiones podemos extraer de estos resultados: en primer lugar a nivel intrapersonal, y como numerosos trabajos han mostrado, existe una clara relación entre los niveles de ansiedad y evitación de cada miembro de la pareja y su propia experiencia relacional, de forma que la seguridad y confianza en la relación, así como la comodidad con la intimidad y proximidad que cada uno percibe se asocia de forma positiva con la calidad de la relación que esa misma persona experimenta (Holland, Fraley, y Roisman, 2012; Lowyck et al., 2008; Mikulincer et al., 2002; Mikulincer y Shaver, 2007; Molero, et al., 2011). En este sentido, cabe destacar el rol más relevante que, en nuestro estudio, parece jugar la propia evitación, algo que también han corroborado otros trabajos (Kane, Jaremka, Guichard, Ford, Collins y Feeney, 2007; Molero, et al., 2011).

En segundo lugar, a nivel interpersonal, cabe destacar el efecto negativo de la ansiedad del varón respecto a la calidad de la relación percibida por parte de la mujer. En este caso, nuestros datos no concuerdan con los hallados en otras investigaciones, en las que era la ansiedad de la mujer la que se asociaba de forma más clara con la menor calidad relacional percibida por el varón (Kane et al., 2007; Kirkpatrick y Davis, 1994). Aunque pueden ser múltiples las razones, nos parece posible pensar que en nuestro contexto, en el que se espera del varón una mayor fortaleza y seguridad, la mujer tenga una experiencia relacional más positiva con una pareja que se encuentra segura en la relación y, que por el contario, la inseguridad del varón y la continua necesidad de reaseguración, típica de la persona más ambivalente, pueda hacer que ellas se sientan más a disgusto. Es factible también que la propia composición de la muestra pueda condicionar esta asociación; en concreto la heterogeneidad en la duración de la relación (de dos a ochenta y cuatro meses), ya que es posible que en las relaciones de corta duración los niveles de ansiedad sean mayores, debido a la inseguridad derivada de la todavía precaria estabilidad de la relación. En este sentido, Heffernan, et al., (2012) han encontrado recientemente que a medida que aumenta la duración de la relación, es más probable que las personas busquen en sus parejas las funciones que cumple el apego. Obviamente, se hacen necesarios otros 
trabajos que nos permitan clarificar esta asociación y las posibles causas de ella.

En relación al último objetivo, pretendíamos comprobar el rol mediador del apego adulto entre el apego en la infancia y la calidad de la relación. Tal y como planteamos en la última de las hipótesis, observamos una conexión entre la vinculación afectiva con los padres durante la infancia y la calidad percibida en la relación de pareja, pero esa conexión estaba mediatizada por la Ansiedad-Evitación en la relación actual. Es decir, el apego a los padres en términos de base segura y refugio tiene un efecto indirecto en la calidad de la relación de pareja de los jóvenes adultos (lo haría a través del apego adulto). Por lo tanto, la percepción de bajos niveles de apoyo y seguridad parental puede favorecer un modelo de relación de pareja caracterizado también por carencias en términos de confianza, seguridad, intimidad, conexión y proximidad, que a su vez, tendrían un efecto directo en una menor calidad de la relación, y al contrario.

Estos resultados avalan la importancia de profundizar en el conocimiento de nuevos factores que pueden actuar de mediadores entre la vinculación afectiva en la infancia y las relaciones de pareja adultas (Scharf y Mayseless, 2001; Simpson, Collins, Tran y Haydon, 2007).

\section{Conclusiones}

\section{Referencias}

Ainsworth, M. D. S. (1991). Attachments and other affectional bonds across the life cycle. En C. M. Parkes, J. Stevenson-Hinde, y P. Marris (Eds.), Attachment across the life cycle (pp.122-149). Londres: Routledge.

Ainsworth, M. D. S., Blehar, M. C., Waters, E. y Wall, S. (1978). Patterns of attachment: apsychological study of the strange situation. Hillsdalle: Lawrence Erlbaum.

Alonso-Arbiol, I., Balluerka, N. y Shaver, P. R. (2007). A Spanish version of the Experiences in Close Relationships (ECR) adult attachment questionnaire. Personal Relationships, 14, 45-63. DOI: 10.1111/j.14756811.2006.00141.x

Auslander, B. A., Short, M. B., Succop, P. A., y Rosenthal, S. L. (2009). Associations between parenting behaviors and adolescent romantic relationships. Journal of Adolescent Health, 45(1), 98-101.

Bowlby, J. (1969). Attachment and loss: Vol. 1. Attachment. New York: Hogarth Press.

Bowlby, J. (1982). Attachment and loss: retrospect and prospect. American Journal of Orthopsychiatry, 52, 664-678.

Bucx, F., y SeiffgeKrenke, I. (2010). Romantic relationships in intra-ethnic and inter-ethnic adolescent couples in Germany: The role of attachment to parents, self-esteem, and conflict resolution skills. International Journal of Behavioral Development, 34(2), 128-135. DOI: 10.1177/0165025409360294.

Collins, W. A., y Dulmen, M. (2006). "The course of true love(s)...": Origins and pathways in the development of romantic relationships. In A. C. Crouter, y A. Booth (Eds.), Romance and sex in adolescence and emerging adulthood (pp. 63-86). Mahwah, NJ: Lawrence Erlbaum.

Collins, W. A., Welsh, D. P., y Furman, W. (2009). Adolescent romantic relationships. Annual Review of Psychology, 60, 631-652. DOI: 10.1146/annurev.psych.60.110707.163459.

Conger, R. D., Cui, M, Bryant, C. M. y Elder, G. H. Jr. (2000).Competence in early adult romantic relationships: A developmental perspective on family influences. Journal of Personality and Social Psychology, 79, 224-237
En resumen, los resultados del trabajo ponen de manifiesto asociaciones significativamente positivas entre la vinculación afectiva en la infancia y la calidad de la relación, comprobándose además el efecto mediador del apego actual. Además, también aparecieron algunas diferencias entre los sexos: el padre del sexo opuesto parece que jugaría un rol más importante que el propio en los procesos afectivos. Por último, se encontraron asociaciones negativas entre la ansiedad y evitación de cada miembro de la pareja y su propia experiencia relacional, y en algún caso, con la experiencia relacional del otro.

Somos conscientes de que muchas de las interpretaciones ofrecidas aquí deben contrastarse en mayor medida, y que la calidad de la relación de pareja depende de otros muchos factores intra e interpersonales. A pesar de las limitaciones del estudio, como la representatividad de la muestra, o la evaluación a través del uso de autoinformes (no siempre el método más válido en la evaluación de este tipo de procesos, - ver Scharf y Mayseless, 2008-.) creemos que nuestro trabajo contribuye en algún grado al conocimiento del papel que juegan los procesos afectivos infantiles y actuales en el ajuste y calidad de las relaciones de pareja de jóvenes adultos.

Agradecimientos.- Este trabajo se ha realizado gracias al proyecto de investigación financiado por el MICINN (Ministerio de Ciencia e Innovación), con referencia PSI2009-10826.

Crockett, L. J., y Randall, B. A. (2006). Linking adolescent family and peer relationships to the quality of young adult romantic relationships: The mediating role of conflict tactics. Journal of Social y Personal Relationships, 25(5), 761-780. DOI: $10.1177 / 0265407506068262$.

Delgado, I., Oliva, A. y Sánchez-Queija, I (2011). Apego a los iguales durante la adolescencia y la adultez emergente. Anales de Psicología, 27, 155163.

Dobson, J. C. (2005). Preparing for adolescence: family guide and workbook (Rev. ed.). Ventura, Calif.: Gospel Light.

Donnellan, M. B., Larsen-Rife, D., y Conger, R. D. (2005). Personality, Family History, and Competence in Early Adult Romantic Relationships. Journal of Personality y Social Psychology, 88(3), 562-576. DOI: 10.1037/0022-3514.88.3.562.

Doyle, A. B., Brendgen, M., Markiewicz, D., y Kamkar, K. (2003). Family Relationships As Moderators of the Association Between Romantic Relationships and Adjustment in Early Adolescence. Journal of Early Adolescence, $23(3), 316$.

Doyle, A. B., Lawford, H., y Markiewicz, D. (2009). Attachment style with mother, father, best friend, and romantic partner during adolescence. Journal of Research on Adolescence, 19(4), 690-714. DOI: 10.1111/j.15327795.2009.00617.x

Feeney, J. A. (1999) Adult romantic attachment and couple relationships. In J. Cassidy and P. R. Shaver (Eds.), Handbook of Attachment: Theory, Research, and Clinical Applications. New York: The Guilford Press.

Fraley R. C. (2002). Attachment stability from infancy to adulthood: Metaanalysis and dynamic modeling of developmental mechanisms. Personality and Social Psychology Review, 6, 123-151.

Fraley, R. C., Heffernan, M. E., Vicary, A. M., y Brumbaugh, C. C. (2011). The Experiences in Close Relationships-Relationship Structures Questionnaire: A method for assessing attachment orientations across relationships. Psychological Assessment, 23, 615-625. DOI: 10.1037/a0022898. 
Fraley, R. C., Waller, N. G., y Brennan, K. A. (2000). An item response theory analysis of self-report measures of adult attachment. Journal of Personality and Social Psychology, 78, 350-365.

Furman, W. y Buhrmester, D. (2009). The Network of Relationships Inventory: Behavioral Systems Version, International Journal of Behavioral Development, 33, 470-478. DOI: 10.1177/0165025409342634.

Furman, W., Ho, M. H. y Low, S.M. (2008). The rocky road of adolescent romantic experience: dating and adjustment. In R. C. M. E. Engels, M. Kerr, H. Stattin, (Eds.), Friends, Lovers, and Groups: Key Relationships in Adolescence, (pp. 61-80). New York: Wiley.

Furman, W. y Winkles, J. K. (2010). Predicting romantic involvement, relationship cognitions, and relationship qualities from physical appearance, perceived norms, and relational styles regarding friends and parents. Journal of Adolescence, 33, 827-836. DOI: 10.1016/j.adolescence.2010.07.004.

Giuliani, C., Iafrate, R., y Rosnati, R. (1998). Peer-Group and Romantic Relationships in Adolescents from Intact and Separated Families. Contemporary Family Therapy: An International Journal, 20(1), 93-105.

Gómez-Zapiain, J., Ortiz, M. J. y Gómez-Lope, J. (2011). Experiencia sexual, estilos de apego y tipos de cuidados en las relaciones de pareja. Anales de Psicología, 27, 447-456.

Ha, T., Overbeek, G., de Greef, M., Scholte, R. H. J., y Engels, R. C. M. E. (2010). The importance of relationships with parents and best friends for adolescents' romantic relationship quality: Differences between indigenous and ethnic dutch adolescents. International Journal of Behavioral Development, 34(2), 121-127. DOI: 10.1177/0165025409360293.

Hazan, C., y Shaver, P. (1987). Romantic love conceptualized as an attachment process. Journal of Personality and Social Psychology, 52, 511-524.

Holland, A. S., Fraley, R. C. y Roisman, G. I. (2012). Attachment styles in dating couples: Predicting relationship functioning over time. Personal Relationships, 19, 234-246. DOI: 10.1111/j.1475-6811.2011.01350.x.

Heffernan, M. E., Fraley, R. C., Vicary, A. M., y Brumbaugh, C. C. (2012). Attachment features and functions in adult romantic relationships. Journal of Social and Personal Relationships, 29, 671-693. DOI: $10.1177 / 0265407512443435$.

Kane, H. S., Jaremba, L. M., Guidarch, A.C. Ford, M. B., Collins, N.L., y Feeney, B.C. (2007). Feeling supported and feeling satisfied: How one partner's attachment style predicts the other partner's relationship experiences. Journal of Social and Personal Relationships, 24(4), 535-555. DOI: 10.1177/0265407507079245.

Kirkpatrick, L. A., y Davis, K. E. (1994). Attachment style, gender, and relationship stability: A longitudinal analysis. Journal of Personality and Social Psychology, 66(3), 502-512.
Leadbeater, B. J., Banister, E. M., Ellis, W. E., y Yeung, R. (2008). Victimization and relational aggression in adolescent romantic relationships: The influence of parental and peer behaviors, and individual adjustment. Journal of Youth and Adolescence, 37(3), 359-372. DOI: 10.1007/s10964007-9269-0.

Lowyck, B., Luyten, P., Demyttenaere, K., y Corveleyn, J. (2008). The role of romantic attachment and self-criticism and dependency for the relationship satisfaction of community adults. Journal of Family Therapy, 30(1), 78-95. DOI: 10.1111/j.1467-6427.2008.00417.x.

McHale, S. M., Crouter, A. C., y Whiteman, S. D. (2003). The family contexts of gender development in childhood and adolescence. Social Development,12, 125-148.

Mikulincer, M., Florian, V., Cowan, P. A. y Cowan, C. P. (2002). Attachment security in couple relationships: a systemic model and its implications for family dynamics. Family Process, 41, 405-434.

Mikulincer, M. y Shaver, P. R. (2003). The attachment behavioral system in adulthood: Activation, psychodynamics, and interpersonal processes. In M. P. Zanna, (Ed.), Advances in experimental social psychology, Vol. 35. (pp. 53-152) Academic Press: New York.

Mikulincer, M. y Shaver, P. R. (2007). Attachment in adulthood: Structure, dynamics, and change. Guilford Press: New York.

Molero, F., Shaver, P. R., Ferrer, E., Cuadrado, I. y Alonso-Arbiol, I. (2011). Attachment insecurities and interpersonal processes in Spanish couples: A dyadic approach. Personal Relationships, 18, 617-629. DOI: 10.1111/j.1475-6811.2010.01325.x.

Scharf, M., y Mayseless, O. (2001).The capacity for romantic intimacy: Exploring the contribution of best friend and marital and parental relationships. Journal of Adolescence, 24, 379-399.

Scharf, M., y Mayseless, O. (2008). Late adolescent girls' relationships with parents and romantic partner: The distinct role of mothers and fathers. Journal of Adolescence, 31, 837-855. DOI: 10.1016/j.adolescence.2008.06.012.

Seiffge-Krenke, I., Overbeek, G., y Vermulst, A. (2010). Parent-child relationship trajectories during adolescence: Longitudinal associations with romantic outcomes in emerging adulthood. Journal of Adolescence, 33, 159-171. DOI: 10.1016/j.adolescence.2009.04.001.

Simpson, J. A., Collins, W. A., Tran, S., y Haydon, K.C. (2007). Attachment and the experience and expression of emotions in romantic relationships: A developmental perspective. Journal of Personality and Social Psychology, 92, 355-367.

SPSS 18 para Windows (2010). Release 18.0.2. SPSS y IBM Company: Chicago.

(Articulo recibido: 08-09-2011; revisado: 26-03-2013; aceptado: 04-04-2013) 
ANEXO I. Muestra de ítems del NRI-Behavioral Systems Version (NRI-BSV) (Furman y Buhrmester, 2009)

Base Segura de la Madre y del Padre ${ }^{1}$

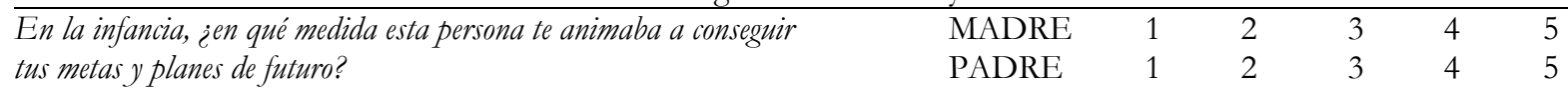

Refugio de la Madre y del Padre ${ }^{1}$

En la infancia, ¿en qué medida esta persona te animaba a intentar

$\begin{array}{llllll}\text { MADRE } & 1 & 2 & 3 & 4 & 5\end{array}$

cosas nuevas que querías hacer pero que te daban miedo?

$\begin{array}{llllll}\text { PADRE } & 1 & 2 & 3 & 4 & 5\end{array}$

${ }^{1}$ Rango: de 1 (poco o nada) a 5 (extremadamente mucho)

ANEXO II. Muestra de ítems del Experiences in Close Relationships-Revised (ECR-R)

(Fraley, Waller y Brennan, 2000)

\begin{tabular}{lllllllll}
\hline \multicolumn{1}{c}{ Ansiedad $^{1}$} & \\
\hline No me preocupa mucho que mi pareja me deje & 1 & 2 & 3 & 4 & 5 & 6 & 7 & \\
Cuando muestro mis sentimientos a mi pareja, tengo miedo de que no sienta lo mismo por mi & 1 & 2 & 3 & 4 & 5 & 6 & 7 & 7 \\
Mi relación me genera mucha preocupación & 1 & 2 & 3 & 4 & 5 & 6 & 7 \\
\hline
\end{tabular}

Evitación $^{1}$

Me siento cómodo/ a compartiendo mis pensamientos y sentimientos con mi pareja

Prefiero no tener demasiada cercanía e intimidad emocional con mi pareja

Le cuento a mi pareja prácticamente todo

$\begin{array}{lllllll}1 & 2 & 3 & 4 & 5 & 6 & 7\end{array}$

${ }^{1}$ Rango: de 1 (totalmente en desacuerdo) a 7 (totalmente de acuerdo) 